\title{
Association between pro-inflammatory alleles and allergic phenotypes in Xhosa adolescents
}

Craig Laurence, Lize van der Merwe, Guicheng Zhang, Peter Le Souëf and Michael Levin

\begin{abstract}
Background: Significant differences exist in the prevalence, spectrum, and severity of allergic diseases between developing and developed countries and between subpopulations within single countries. These discrepancies likely result from a complex interaction between genetic and environmental factors. However, the precise nature of the contribution of ethnicity to genetic differences in the predisposition to allergic disease is not yet fully understood. In particular, there is a paucity of literature regarding the genetic determinants of allergic disease in people of black African origin with little or no genetic admixture.

Objective: We aimed to analyze associations between 27 single nucleotide polymorphisms (SNPs) and allergy phenotypes in the local Xhosa population.

Methods: A convenience sample of 213 Xhosa teenagers was enrolled at a local high school. Phenotypic data were collected in the form of a symptom questionnaire, skin prick tests for common food and aeroallergens, total serum IgE, and IgE to Ascaris lumbricoides. In addition, genotyping was performed to establish the prevalence of putative pro-inflammatory alleles.
\end{abstract}

Results: We demonstrated several significant associations between polymorphisms and allergy phenotypes. In particular, 2 polymorphisms in the IL-10 gene (IL10 -592A>C and IL10 $-1082 A>G$ ) and 1 in the IL-4 gene (IL4 $-589 \mathrm{C}>\mathrm{T}$ ) showed multiple associations with allergic sensitization and asthma phenotypes. Other polymorphisms, across a multitude of genes with discrepant functions, showed less consistent associations.

Conclusion: This study represents an important first step in genotype/phenotype association in this population. Further research is required to confirm or refute our findings.

\section{1 | Introduction}

Asthma and other allergic illnesses likely result from a complex interaction between genetic susceptibility and environmental exposure. Differences in prevalence, spectrum, severity, and management of allergic diseases between and within populations result in significant discrepancies in morbidity 1,2 and mortality.3,4 Several groups have shown that whilst the prevalence of respiratory allergies in people of black African origin is lower than in European populations, they suffer disproportionate morbidity ${ }^{1,2}$ and mortality.5,6 


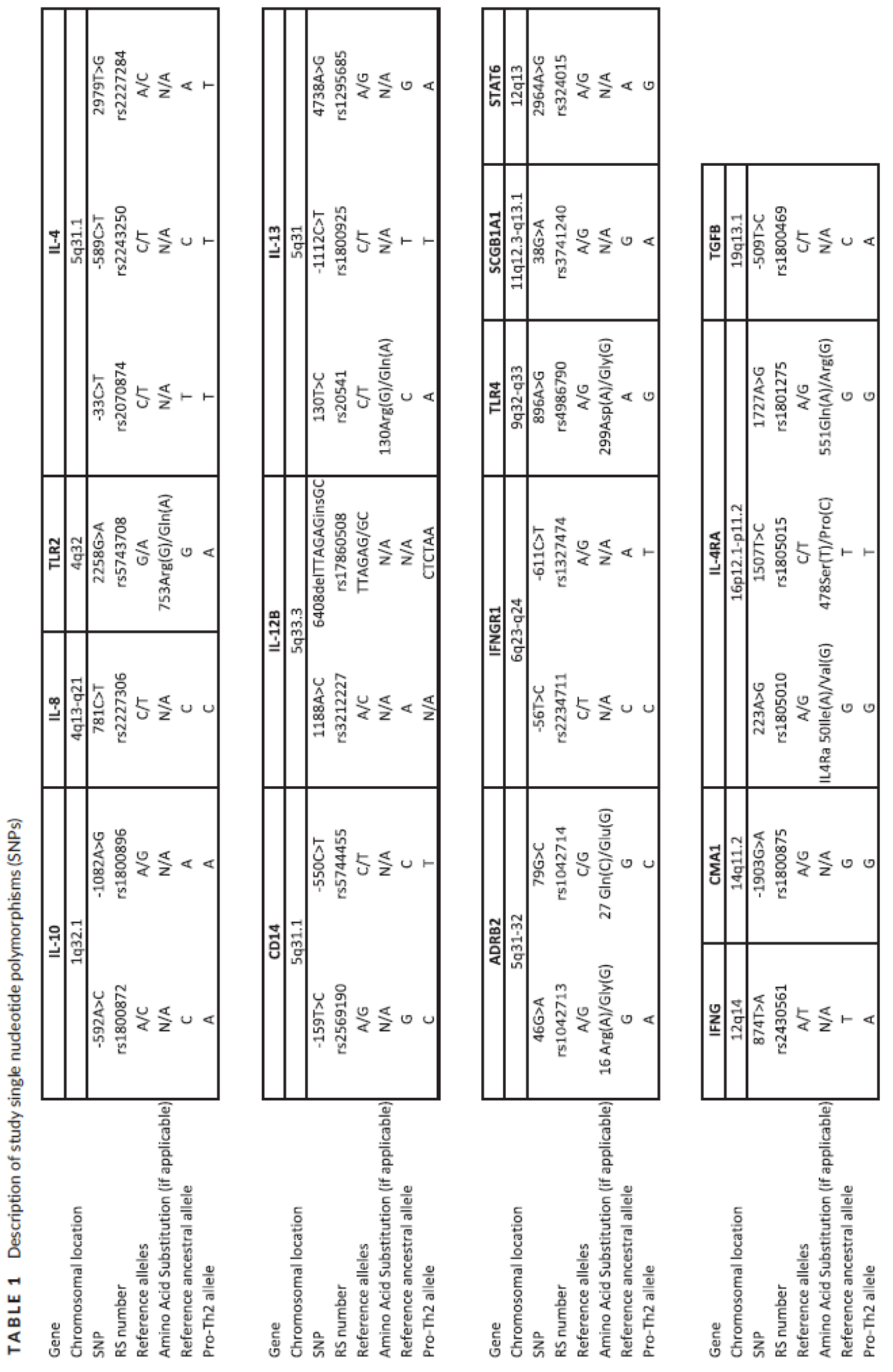


Although several polymorphisms have shown associations in genomewide association studies (GWAS) in the developed world, there is a paucity of similar data from the developing world, and for people of black African origin in particular, where little admixture has occurred.7 Some evidence suggests that susceptibility loci may be different for people of differing ethnicities. 8 Difficulties in unbundling ethnicity from accompanying social and economic constructs make it extremely challenging to assess the contribution of each in the disproportionate morbidity and mortality from allergic disease in people of certain ethnicities. 9 However, it seems likely that genetic determinants do play a role in ethnic differences in the prevalence and phenotypic characteristics of allergic disease. ${ }^{10}$

As in many other developing countries, the prevalence of allergic disease in South Africa seems to be increasing. ${ }^{11}$ Allergic disease is more prevalent in urban than rural populations and in white than black African subjects; however, the gradient ${ }^{12}$ between these populations has narrowed over time.13,14 Additionally, there has been a disproportionate increase in both house dust mite (HDM) sensitization and bronchial hyper-responsiveness (BHR) in recently urbanized children of black African origin. ${ }^{13,14}$ These factors have led local authors to hypothesize that adoption of a Western lifestyle has been partly causal in the development of allergic disease in black South Africans ${ }^{13}$; however, the magnitude of this contribution remains to be interrogated in Africa. The high prevalence and severity of allergic diseases amongst black African subjects in high-income countries 6,15 may reflect unique genetic susceptibility to allergic disease. ${ }^{16}$ A plausible explanation for this situation is that Africans' genotypes reflect their evolutionary adaptation toward strong pro-Th2 immune responses that were required to combat the hostile helminthic infections endemic in the more tropical African environment and that these pro-Th2 genotypes produce their pro-allergic genetic makeup. ${ }^{17}$ It follows that these pro-Th2 genotypes would have been evolutionally less important or indeed deleterious for humans who left Africa and gradually reduced in frequency. ${ }^{17}$ In addition, current helminthic infection has been shown to reduce skin prick reactivity and may reduce allergic symptoms through the production of blocking antibodies

or other parasite-induced mechanisms predominantly aimed at helminth survival. ${ }^{18}$ Ascaris lumbricoides is one of the most prevalent helminth infections found in humans and has been estimated to affect over 100 million people in sub-Saharan Africa alone. ${ }^{19}$

In this study, we aimed to interrogate genetic susceptibility to asthma and allergic disease in black South Africans by evaluating the relationship between pro-inflammatory alleles (PIAs) and clinical and laboratory phenotypes of allergic disease in the Xhosa population.

\section{2 | Methods}

A convenience sample of 213 Xhosa children, with a mean age of 17 years, was obtained from an entire grade 11 class in a high school in Cape Town, between May and September 2005. Children 
were identified as Xhosa if they indicated that both parents spoke Xhosa as a first language. The children completed a questionnaire modified from the International Study of Asthma and Allergies in Childhood (ISAAC) regarding symptoms of asthma, eczema, and rhinitis. Skin prick tests (SPTs) to common aero- and food allergens (all ALK-Abello ${ }^{\circledR}$ except for potato, which was a freshly prepared extract), blood samples for total IgE and $A$. lumbricoides-specific IgE, and a modified methacholine challenge for BHR were performed. The method for this modified challenge has been previously published 14 by our group and is based on the Protocol by Yan et al. 20

Blood samples were taken for genotyping of twenty-seven single nucleotide polymorphisms (SNPs) on sixteen distinct genes (see online repository for full description of SNPs) (Table 1). These SNPs were chosen based on previous descriptions in the literature of association with allergic phenotypes at a population level, as well as their occurrence in genes known or theorized to be associated with allergy and/or asthma. These genes, broadly speaking, fitted into one of several categories:

1. Genes associated with anti-inflammatory immune responses or the development of immune-tolerance (IL-10; TGF- $\beta$; SCG- $\beta 1 \mathrm{~A} 1$ ) Genes associated with a TH1-predominant immune response (IL-8; IL-12 $\beta$; IFN- $\gamma$; CD14; TLR2; TLR4) Genes associated with a TH2predominant immune response (IL-4; IL-4Ra; IL-13; STAT6; CMA1) Genes associated with allergic bronchospasm (ADBR2)

Genotyping was conducted by the Australian Genome Research Facility, and the genotyping method has been previously published in this journal. ${ }^{21}$

\subsection{Ethical considerations}

Ethical approval was obtained via the University of Cape Town Human Research Ethics Committee (HREC 109/2001 and 335/2014). Written consent for the study was obtained from the children and their parents, the school, and the department of education.

\subsection{Statistical analysis}

All statistical analysis was completed using R. ${ }^{22}$ We assessed genotype and allele distribution, using the genetics package from $\mathrm{R}, 23$ the number of individuals successfully genotyped and performed an exact test of Hardy-Weinberg equilibrium on all SNPs. Logistic regression was used to look for evidence of association between the twenty-seven dichotomous SNPs and clinical and laboratory markers of allergy. All models were corrected for several exposures (age, gender, cat, dog or farm animal exposure, cigarette or fire smoke exposure and type of accommodation), by adding them to the models as fixed effects. We modeled genotype (three genotypes, conferring two degrees of freedom) and additive allelic (counting the number of minor alleles to determine a "dose-response") predictor representations of each SNP. The results guided us to find the best fitting model (lowest $P$-value) from the five possibilities: genotype, additive allelic, dominant, recessive, and heterozygote. All $P$-values, 
effect sizes (measured as change in odds \%), and confidence intervals were derived from these models.

TABLE 2 Summary of effects of genetic associations

\begin{tabular}{|c|c|c|c|c|}
\hline \multirow[t]{2}{*}{ SNP } & \multirow[t]{2}{*}{ Phenotype } & \multicolumn{3}{|l|}{ Inheritance } \\
\hline & & Model & Effect Type & Magnitude of Effect (\%) \\
\hline \multirow[t]{5}{*}{ IL10 $-592 A>C$} & Positive Der p SPT & Additive allelelic & Each $\mathrm{A}$ allele changes odds by & $109(95 \% \mathrm{Cl}: 21$ to 267$)$ \\
\hline & Positive Der f SPT & Additive allelelic & Each $\mathrm{A}$ allele changes odds by & $101(95 \% \mathrm{Cl}: 15$ to 256$)$ \\
\hline & Any positive HDM SPT & Additive allelelic & Each $\mathrm{A}$ allele changes odds by & $86(95 \% \mathrm{Cl}: 9$ to 221$)$ \\
\hline & Eczema ever & Additive allelelic & Each $A$ allele changes odds by & $-43(95 \% \mathrm{Cl}:-66$ to -3$)$ \\
\hline & Eczema in past year & Additive allelelic & Each $\mathrm{A}$ allele changes odds by & $-46(95 \% \mathrm{Cl}:-69$ to -8$)$ \\
\hline \multirow[t]{8}{*}{ IL10 $-1082 A>G$} & Any positive SPT & Additive allelelic & Each $\mathrm{G}$ allele changes odds by & $-43(95 \% \mathrm{Cl}:-67$ to -4$)$ \\
\hline & Positive Der p SPT & Additive allelelic & Each $\mathrm{G}$ allele changes odds by & $-47(95 \% \mathrm{Cl}:-72$ to -6$)$ \\
\hline & Positive Der $\mathrm{f}$ SPT & Additive allelelic & Each $\mathrm{G}$ allele changes odds by & $-56(95 \% \mathrm{Cl}:-78$ to -19$)$ \\
\hline & Any positive HDM SPT & Additive allelelic & Each $\mathrm{G}$ allele changes odds by & $-57(95 \% \mathrm{Cl}:-77$ to -22$)$ \\
\hline & Any positive aeroallergen SPT & Additive allelelic & Each $\mathrm{G}$ allele changes odds by & $-50(95 \% \mathrm{Cl}:-71$ to -15$)$ \\
\hline & Wheeze in past year & Recessive & $G G$ vs $A A+A G$ & $-87(95 \% \mathrm{Cl}:-99$ to -31$)$ \\
\hline & Self-reported rhinitis & Additive allelelic & Each $\mathrm{G}$ allele changes odds by & $-48(95 \% \mathrm{Cl}:-68$ to -16$)$ \\
\hline & $\mathrm{BHR}$ & Additive allelelic & Each $\mathrm{G}$ allele changes odds by & $-54(95 \% \mathrm{Cl}:-79$ to -8$)$ \\
\hline \multirow[t]{6}{*}{ IL4 $-589 \mathrm{C}>\mathrm{T}$} & Any positive SPT & Additive allelelic & Each $\mathrm{C}$ allele changes odds by & $-47(95 \% \mathrm{Cl}:-70$ to -8$)$ \\
\hline & Positive Cockroach SPT & Additive allelelic & Each $C$ allele changes odds by & $-54(95 \% \mathrm{Cl}:-78$ to -9$)$ \\
\hline & Any positive aeroallergen SPT & Additive allelelic & Each $\mathrm{C}$ allele changes odds by & $-44(95 \% \mathrm{Cl}:-68$ to -3$)$ \\
\hline & Ever wheezed & Heterozygote & TC vs both homozygotes & $-67(95 \% \mathrm{Cl}:-84$ to -34$)$ \\
\hline & Wheeze in past year & Heterozygote & TC vs both homozygotes & $-65(95 \% \mathrm{Cl}:-84$ to -27$)$ \\
\hline & Ever eczema & Additive allelelic & Each $\mathrm{T}$ allele changes odds by & $79(95 \% \mathrm{Cl}: 3$ to 215$)$ \\
\hline \multirow{2}{*}{$\begin{array}{l}\text { IL12B } \\
\text { 6408delTTA- } \\
\text { GAGinsGC }\end{array}$} & Any positive food SPT & Dominant model & Any TTAGAG vs GC/GC & $305(95 \% \mathrm{Cl}: 31$ to 1374$)$ \\
\hline & Ever wheeze & Recessive & TTAGAG/TTAGAG vs other two & $-87(95 \% \mathrm{Cl}:-99$ to -34$)$ \\
\hline \multirow[t]{3}{*}{ IL13 130T >C } & Any positive food SPT & Additive allelelic & Each $\mathrm{T}$ allele changes odds by & $-74(95 \% \mathrm{Cl}:-95$ to -3$)$ \\
\hline & Self-reported rhinitis & Recessive & $\mathrm{TT}$ vs $\mathrm{CC}+\mathrm{CT}$ & $192(95 \% \mathrm{Cl}: 6$ to 807$)$ \\
\hline & Self-reported eczema & Heterozygote & $\mathrm{CT}$ vs $\mathrm{CC}+\mathrm{TT}$ & $216(95 \% \mathrm{Cl}: 45$ to 608$)$ \\
\hline$A D B R 246 A>G$ & Any positive aeroallergen SPT & Recessive & $A A$ vs $G G+G A$ & $166(95 \% \mathrm{Cl}: 25$ to 472$)$ \\
\hline IFNGR1 $-56 \mathrm{~T}>\mathrm{C}$ & $\begin{array}{l}\text { Total serum IgE } \\
\text { Any positive food SPT }\end{array}$ & $\begin{array}{l}\text { Additive allelic } \\
\text { Dominant }\end{array}$ & $\begin{array}{l}\% \text { change in IgE per T allele All } 52 \\
\text { with CC had negative SPTs. So } \\
\text { the effect of any } T(T T+C T) \text { is } \\
\text { very strong. But could not be } \\
\text { estimated, because the effect is } \\
\text { indivisible by } 0 \text {. }\end{array}$ & $\begin{array}{l}-30(95 \% \mathrm{Cl}:-48 \text { to }-6) \\
\text { N/A }\end{array}$ \\
\hline STAT6 $2964 A>G$ & Self-reported eczema & Dominant & $\mathrm{GA}+\mathrm{AA}$ vs $\mathrm{GG}$ & $-67(95 \% \mathrm{Cl}:-90$ to -12$)$ \\
\hline IL4RA $1507 \mathrm{~T}>\mathrm{C}$ & Ever wheezed & Additive allelelic & Each $\mathrm{C}$ allele changes odds by & $69(95 \% \mathrm{Cl}: 1$ to 188$)$ \\
\hline \multirow[t]{2}{*}{ IL $4 R A$ A $1727 A>G$} & Ever wheezed & Dominant & $\mathrm{GA}+\mathrm{AA}$ vs $\mathrm{GG}$ & $-55(95 \% \mathrm{Cl}:-79$ to -8$)$ \\
\hline & Wheeze in past year & Dominant & $\mathrm{GA}+\mathrm{AA}$ vs $\mathrm{GG}$ & $-58(95 \% \mathrm{Cl}:-83$ to -6$)$ \\
\hline
\end{tabular}

BHR, bronchial hyper-responsiveness; HDM, house dust mite; IgE, Immunoglobulin E; SPT, skin prick test.

We elected to set our significance level to a $P$-value $<.05$ and not to correct for multiple testing, for several reasons. Firstly, there is no single established method of correcting for multiple testing. 24,25 In addition, because this study is the first to explore these 
polymorphisms in a black African population, we felt that the repercussions of not reporting a positive association would be more significant than reporting a false positive association.

\section{3 | Results}

\subsection{Phenotypic characteristics}

The phenotypic characteristics of the study group have been published previously, $14,15,26$ but are summarized below. Our group comprised 213 individuals, of which 60\% (127) were female. The self-reported prevalence of asthma was 10\% (22), whilst BHR was demonstrated in $16 \%$ (33). The self-reported prevalence of rhinitis was $49 \%$ (103), and the self-reported prevalence of eczema was $21 \%$ (44). There was poor overlap between a self-reported diagnosis of asthma and challenge-demonstrated BHR, with only 40\% (8) of individuals reporting symptoms of asthma demonstrating BHR. Asthma was strongly associated with eczema and positive aeroallergen skin prick tests, whereas bronchial hyper-responsiveness was strongly associated with positive aeroallergen skin prick tests, and weakly associated with rhinitis.

Skin prick tests and other tests for sensitization perform well in the detection of both aeroallergen and food sensitization in black African subjects, unless there are confounders such as severe parasite infestation. ${ }^{26}$ The predictive values of these tests are excellent for aeroallergen sensitization, although there is some preliminary evidence that in our setting, the positive and negative predictive values of such tests may be lower for food allergens, particularly in unselected populations such as the one studied here. 27

Total serum IgE levels were associated with atopy and IgE to A. lumbricoides. ${ }^{26}$ IgE to $A$. lumbricoides was strongly associated with aeroallergen sensitization and BHR, but not with asthma, rhinitis, or eczema. 15

\subsection{Genetic characteristics and associations}

All SNPs genotyped were in Hardy-Weinberg Equilibrium. The genotype, allele, and haplotype frequencies, as well as an analysis of linkage disequilibrium, have been included as supplementary information. Several of the SNPs in our study showed associations with laboratory and clinical phenotypes. These associations are listed in Table 2, and a discussion of the significance of these associations follows below.

\section{4 | Discussion}

We demonstrate several significant associations between SNPs and phenotypic markers of allergic disease. The genes and genotypes for which these associations were demonstrated have been previously shown to be important in determining susceptibility to atopy and asthma in other populations ${ }^{28-34}$, but not to date in an African population resident in southern Africa.

The two polymorphisms that displayed the most consistent results were both in the IL-10 gene. The A allele of IL1O -592A $>$ C was associated with significantly elevated odds of HDM 
sensitization; however, there were no associations between this allele and any clinical phenotypes of allergic disease. Zheng et $\mathrm{al}^{28}$ suggested a dominant effect for any Acontaining genotype and asthma in a recent meta-analysis, although on subgroup analysis, this effect was restricted to people of Asian origin, and only in adults. Similar associations were found by Nie et $\mathrm{al}^{29}$ in another recent meta-analysis. Conversely, we found the A allele to be protective against a history of ever having eczema, or of having eczema in the past year. This is in contrast with a study by Sohn et al30 who demonstrated an increased frequency of atopic dermatitis in Korean children with any A-containing genotype (dominant model). This discrepancy may represent a specific gene-gene or geneenvironment interaction in this population, or possibly a type one error.

IL10 -1082A $>\mathrm{G}$ was associated with aeroallergen SPT positivity, and in particular HDM SPT positivity. We found a protective effect of the $G$ allele against aeroallergen sensitization, as well as against clinical asthma and rhinitis phenotypes. In addition, the $\mathrm{G} / \mathrm{G}$ genotype was associated with a decreased odds \% of wheeze. These results are broadly in keeping with results from several meta-analyses, $28,29,31$ although none of these included studies of people of black African origin.

We also demonstrated several interesting results from polymorphisms in genes that are known to promote a $\mathrm{T}_{\mathrm{H}} 2$ immune response. The $\mathrm{C}$ allele of the IL4 $-589 \mathrm{C}>\mathrm{T}$ polymorphism showed decreased odds of having any positive SPT, having any positive aeroallergen SPT, or having a positive cockroach SPT. However, the associations with clinical phenotypes were conflicted, with $\mathrm{T} / \mathrm{C}$ heterozygotes showing decreased odds of ever wheezing, or wheezing in the past year, and the T allele was associated with increased odds of ever having eczema. Any A-containing genotype of the IL-4Ra polymorphism $1727 \mathrm{~A}>\mathrm{G}$ was associated with decreased odds of either ever wheezing, or wheezing in the past year. The literature is conflicted regarding the association of this polymorphism with allergy phenotypes, with several meta-analyses32,33,34showing significant associations with asthma phenotypes in people of Asian origin, whilst others 35 have found only a nominally significant association with asthma.

Analysis of associations between the other SNPs in our study and allergy phenotypes produced mixed results. Examples include IL12 6408delTTAGAGinsGC, where TTAGAG was associated with increased odds of having any positive food SPT, but demonstrated some protection against wheeze and eczema symptoms. Likewise, any A-containing genotype in the polymorphism IL13 130T $>$ C showed decreased odds of having any positive food SPT, but the T/T genotype was associated with increased odds of selfreported rhinitis, and T/C heterozygotes showed increased odds of self-reported eczema. With respect to polymorphisms in the $\beta_{2}$ adrenoceptor gene (ADBR2), we did not find any associations between either of the polymorphisms and asthma phenotypes, although surprisingly, there was an association between the A/A genotype of the $46 \mathrm{~A}>\mathrm{G}$ SNP and having any positive aeroallergen SPT. It is difficult to propose a pathophysiological 
mechanism for this association, and it is questionable whether this represents a true association.

The lack of overlap between bronchial hyper-responsiveness and self-reported symptoms of asthma may in part be explained by different phenotypes of asthma, but also highlights possible methodological concerns in the diagnosis of bronchial hyper-responsiveness (particularly, the use of a handheld glass nebulizer rather than a dosimeter) and/or in the diagnosis of asthma. As previously mentioned, relying on a self-reported diagnosis of asthma rather than a physician diagnosis may not accurately estimate the true prevalence of asthma. ${ }^{11}$ Additionally, language differences and cultural differences in the terminology and understanding of health and disease 36 may have affected prevalence data and other self-reported clinical phenotypes. As these self-reported phenotypes represent the majority of the genetic interactions uncovered in our study, many of these associations would require reproduction in further studies before drawing any firm conclusions. As the Xhosa population, and black African populations in general, has received so little attention with regard to their genetic risks for allergic disease, it is possible that the polymorphisms that confer predisposition to allergic phenotypes in this population may differ from those in people of admixed black African ethnicities. This is of particular importance as gene-gene interactions and gene-environment interactions may mask such associations in other settings.

This study therefore represents an important first step in the analysis of the contribution of genetic risk to allergy phenotypes in people of black African ancestry. Of particular, interest is the associations between polymorphisms in the IL-10 gene, as well as the IL-4 gene, and aeroallergen sensitization.

\section{Conflict of interests}

The research team received grants from the Allergy Society of South Africa (ALLSA) and the School of Child and Adolescent Health (SCAH), University of Cape Town. Neither of the funding sources had any input with regard to the study design; in the collection, analysis, and interpretation of data; in the writing of the report; and in the decision to submit the article for publication. The researchers acted independently of the funders (ALLSA and $\mathrm{SCAH})$.

\section{ORCID}

Craig Laurence http://orcid.org/oooo-0002-8780-2297 


\section{References}

1. Lai CKW, Beasley R, Crane J, Foliaki S, Shah J, Weiland S. Global variation in the prevalence and severity of asthma symptoms: phase three of the International Study of Asthma and Allergies in Childhood (ISAAC). Thorax. 2009;64:476-483.

2. Aït-Khaled N, Pearce N, Anderson HR, Ellwood P, Montefort S, Shah J. Global map of the prevalence of symptoms of rhinoconjunctivitis in children: the International Study of Asthma and Allergies in Childhood (ISAAC) phase three. Allergy. 2009;64:123-148.

3. Masoli M, Fabian D, Holt S, Beasley R. The global burden of asthma: executive summary of the GINA dissemination committee report. Allergy. 2004;59:469478.

4. Arias E, Anderson RN, Kung H-C, Murphy SL, Kochanek KD. Deaths: final data for 2001. Natl Vital Stat Rep. 2003;52:1-115.

5. Akinbami LJ, Schoendorf KC. Trends in childhood asthma: prevalence, health care utilization, and mortality. Pediatrics. 2002;110(2 Pt 1):315-322.

6. Gupta RS, Carrión-Carire V, Weiss KB. The widening black/white gap in asthma hospitalizations and mortality. $J$ Allergy Clin Immunol. 2006;117(2):351-358.

7. Barnes KC. Genomewide association studies in allergy and the influence of ethnicity. Curr Opin Allergy Clin Immunol. 2010;10:427-433.

8. Xu J, Meyers DA, Ober C, et al. Genomewide screen and identification of genegene interactions for asthma-susceptibility loci in three U.S. populations: collaborative study on the genetics of asthma. Am J Hum Genet 2001;68:14371446.

9. Leong $\mathrm{AB}$, Ramsey $\mathrm{CD}$, Celedón JC. The challenge of asthma in mi- nority populations. Clin Rev Allergy Immunol. 2012;43:156-183.

10. Barnes KC, Grant AV, Hansel NN, Gao P, Dunston GM. African Americans with asthma: genetic insights. Proc Am Thorac Soc. 2007;4:58-68.

11. Zar HJ, Ehrlich RI, Workman L, Weinberg EG. The changing preva- lence of asthma, allergic rhinitis and atopic eczema in African adoles- cents from 1995 to 2002. Pediatr Allergy Immunol. 2007;18:560-565.

12. Van Niekerk CH, Weinberg EG, Shore SC, Heese HV, Schalkwyk JV. Prevalence of asthma: a comparative study of urban and rural Xhosa children. Clin Allergy. 1979;9:314-319.

13. Steinman HA, Donson H, Kawalski M, Toerien A, Potter PC. Bronchial hyperresponsiveness and atopy in urban, peri-urban and rural South African children. Pediatr Allergy Immunol. 2003;14:383-393.

14. Levin ME, Muloiwa R, Motala C. Associations between asthma and bronchial hyper-responsiveness with allergy and atopy phenotypes in urban black South African teenagers. S Afr Med J. 2011;101:472-476.

15. Joseph CLM, Williams LK, Ownby DR, Saltzgaber J, Johnson CC. Applying epidemiologic concepts of primary, secondary, and tertiary prevention to the 
elimination of racial disparities in asthma. $J$ Allergy Clin Immunol. 2006;117:233-240.

16. Flores C, Ma S-F, Pino-Yanes M, et al. African ancestry is associated with asthma risk in African Americans. PLoS One. 2012;7:e26807.

17. Le Souëf PN, Goldblatt J, Lynch NR. Evolutionary adaptation of inflammatory immune responses in human beings. Lancet. 2000;356:242244.

18. Levin M, Muloiwa R, Souef PL, Motala C. Ascaris sensitization is as- sociated with aeroallergen sensitization and airway hyperresponsive- ness but not allergic disease in urban Africa. J Allergy Clin Immunol. 2012;130:265-267.

19. de Silva NR, Chan MS, Bundy DA. Morbidity and mortality due to ascariasis: re-estimation and sensitivity analysis of global numbers at risk. Trop Med Int Health. 1997;2(6):519-528.

20.Yan K, Salome C, Woolcock AJ. Rapid method for measurement of bronchial responsiveness. Thorax. 1983;38:760-765.

21. Zhang G, Candelaria P, Mäkelä JM, et al. Disparity of innate immunity- related gene effects on asthma and allergy on Karelia. Pediatr Allergy Immunol. 2011;22:621-630.

22. R Core Team. R: A Language and Environment for Statistical Computing [Internet]. Vienna, Austria: R Foundation for Statistical Computing; 2015. Available from: https://www.R-project.org

23. Warnes G, Gorjanc G, Leisch F, Man M. Population genetics [Internet]. 2013. Available from: https:/CRAN.R-project.org/pack- age=genetics. Accessed March 12, 2017.

24. Hsueh H, Chen JJ, Kodell RL. Comparison of methods for estimating the number of true null hypotheses in multiplicity testing. $J$ Biopharm Stat. 2003;13:675-689.

25. Farcomeni A. A review of modern multiple hypothesis testing, with particular attention to the false discovery proportion. Stat Methods Med Res. 2008; $17: 347-388$.

26. Levin ME, Le Souëf PN, Motala C. Total IgE in urban Black South African teenagers: the influence of atopy and helminth infection. Pediatr Allergy Immunol. 2008;19:449-454.

27. Basera W, Botha M, Gray CL, et al. The South African food sensitisation and food allergy population-based study of IgE-mediated food allergy: validity, safety, and acceptability. Ann Allergy Asthma Immunol. 2015;115:113-119.

28.Zheng XY, Guan WJ, Mao C, et al. Interleukin-10 promoter 1082/- 819/-592 polymorphisms are associated with asthma susceptibility in Asians and atopic asthma: a meta-analysis. Lung. 2014;192:65-73.

29. Nie W, Fang Z, Li B, Xiu QY. Interleukin-10 promoter polymorphisms and asthma risk: a meta-analysis. Cytokine. 2012;60:849-855.

30. Sohn MH, Song JS, Kim K-W, Kim E-S, Kim K-E, Lee JM. Association of interleukin-10 gene promoter polymorphism in children with atopic dermatitis. J Pediatr. 2007;150:106-108. 
31. Hyun $\mathrm{MH}$, Lee $\mathrm{CH}$, Kang $\mathrm{MH}$, Park BK, Lee YH. Interleukin-10 pro- moter gene polymorphisms and susceptibility to asthma: a meta- Analysis. PLoS One. 2013;8:e53758.

32. Nie W, Zang Y, Chen J, Xiu Q. Association between Interleukin-4 Receptor a Chain (IL4RA) I50V and Q551R polymorphisms and asthma risk: an update meta-analysis. PLoS One. 2013;8:e69120.

33. Huang ZY, Cheng BJ, Cai GJ, Zhang BF. Association of the IL-4R Q576R polymorphism and asthma in the Chinese Han population: a meta-analysis. Genet Mol Res (GMR). 2015;14:2900-2911.

34. Loza MJ, Chang B-L. Association between Q551R IL4R genetic vari- ants and atopic asthma risk demonstrated by meta-analysis. J Allergy Clin Immunol. 2007;120:578-585.

35. Murk W, Walsh K, Hsu L-I, Zhao L, Bracken MB, Dewan AT. Attempted replication of 50 reported asthma risk genes identifies a SNP in RAD50 as associated with childhood atopic asthma. Hum Hered. 2011;71:97-105.

36. Levin ME. Different use of medical terminology and culture-specific models of disease affecting communication between Xhosaspeaking patients and Englishspeaking doctors at a South African paediatric teaching hospital. S Afr Med J. 2006;96:1080-1084. 